\title{
Patterned optical anisotropy in woven conjugated polymer systems
}

\author{
C. Müller, ${ }^{1, a)}$ M. Garriga, ${ }^{2}$ and M. Campoy-Quiles ${ }^{2}$ \\ ${ }^{1}$ Department of Chemical and Biological Engineering/Polymer Technology, \\ Chalmers University of Technology, 41296 Göteborg, Sweden \\ ${ }^{2}$ Institut de Ciència de Materials de Barcelona (ICMAB-CSIC), Campus de la UAB, E-08193 \\ Bellaterra, Spain
}

(Received 11 August 2012; accepted 11 October 2012; published online 25 October 2012)

\begin{abstract}
Weaving of highly oriented conjugated polymer/polyethylene tapes is demonstrated to permit the generation of concealed patterns that can be detected under appropriate polarized light illumination. This is achieved by exploiting the fact that the amount of transmitted light varies with the superposition sequence of semi-transparent objects that feature a high degree of linear birefringence as well as linear dichroism. An analysis based on Müller calculus provides a theoretical description of the observed optical behavior. (c) 2012 American Institute of Physics. [http://dx.doi.org/10.1063/1.4764518]
\end{abstract}

Anisotropic optical characteristics of conjugated polymers have been exploited in a broad variety of applications, including polarized-light emitting diodes ${ }^{1-4}$ and optical filters, such as electrochromic, ${ }^{5}$ photoluminescent ${ }^{6-8}$ as well as infrared polarizers, ${ }^{9}$ for use in counterfeiting products, ${ }^{10}$ liquid crystal displays, ${ }^{11}$ and the like. An efficient method to realize such anisotropic optical features is based on unidirectional alignment of the conjugated species in a polymeric host by tensile drawing. ${ }^{6-19}$ However, creation of patterned features, which are a prerequisite for the realization of more complex articles, has so far been challenging when employing this procedure. Principally, this is due to the required mechanical deformation step that prevents the use of regular lithography or printing techniques. Indeed, it seems that selective photobleaching of a photoluminescent moiety embedded in an ultra-high molecular-weight polyethylene (UHMW-PE) matrix is one of the only methods that has been advanced, which permits fabrication of well-defined structures and images in discrete tensile-oriented polymer films. ${ }^{16}$

We explored, therefore, other options to create optical patterns, focusing on superimposing highly anisotropic materials that feature both linear birefringence as well as linear dichroism, produced by standard tensile deformation of a suitable guest/host polymer system. We demonstrate that this approach is compatible with straightforward weaving schemes widely utilized in textile manufacturing. ${ }^{20}$ This is illustrated by the realization of optically concealed images in apparently uniform fabrics (when observed in unpolarized light), which can be detected only under appropriate polarized-light illumination.

We selected blends of regio-regular poly(3-hexylthiophene) (P3HT) and UHMW-PE for the creation of such optically anisotropic systems. P3HT is one of the most extensively studied conjugated polymers, absorbing at wavelengths, $\lambda$, between 400 and $650 \mathrm{~nm}$ in the solid state, ${ }^{21-25}$ whilst UHMW-PE has previously been reported to facilitate uniaxial orientation of high aspect-ratio guest-species by tensile drawing. ${ }^{6-9,11,13-16}$ Samples were prepared by first dissolving $0.01 \mathrm{~g}^{-1}$ P3HT (purchased from Sigma Aldrich;

\footnotetext{
a)e-mail: christian.muller@chalmers.se.
}

$M_{\mathrm{w}} \sim 48 \mathrm{~kg} \mathrm{~mol}^{-1}$; regio-regularity $\left.>90 \%\right)$ and $1 \mathrm{~g} \mathrm{l}^{-1}$ UHMW-PE (obtained from DSM; $M_{\mathrm{w}} \sim 6100 \mathrm{~kg} \mathrm{~mol}^{-1}$ ) in decalin at $130{ }^{\circ} \mathrm{C}$ followed by casting at ambient. Dried 1:99 P3HT/UHMW-PE films were tensile drawn to 30-40 times their initial length at $120^{\circ} \mathrm{C}$ to produce oriented tapes of thickness $d \sim 10 \mu \mathrm{m}$. Highly efficient alignment of the matrix polymer and thus of the conjugated host species in the drawing direction was achieved, giving rise to high levels of linear birefringence (retardance) and linear dichroism (attenuation), respectively. The linear birefringence is readily apparent from a series of cross-polarized optical micrographs in Fig. 1(a). In addition, linear dichroism occurs at wavelengths, at which the P3HT moiety is absorbing, as evidenced by polarized UV-Vis absorbance spectra (recorded with a Perkin Elmer Lambda 900 spectrophotometer) as well as polarized optical micrographs in Fig. 1(b). As a matter of fact, a dark purple color with a maximum absorbance at $550 \mathrm{~nm}$ was found for such aligned tapes when backilluminated with light polarized parallel to the drawing direction, whereas perpendicularly polarized light resulted in a pale pink appearance with a blue-shifted maximum absorbance around $500 \mathrm{~nm}$.

There exists a vast library of fabric patterns that can be generated by weaving the here discussed P3HT/UHMW-PE tapes. Each weave can be considered as an individual element of such a system, comprised of at least two tapes crossing each other at angle $\varphi$, where $\varphi$ is the relative orientation between the drawing directions of the individual tapes. For instance, in case of a plain weave, which features a basketcase like alternating weaving pattern, ${ }^{20}$ the relative orientation of tapes that make up adjacent segments, here labeled $\mathrm{A}$ and $\mathrm{B}$, is equivalent to two pairs of tapes with opposite plaiting sequence so that $\varphi_{\mathrm{A}}=-\varphi_{\mathrm{B}}$. Similar to a single tape, weaves are likely to be of featureless appearance when observed in plain, unpolarized light. However, when placed between a pair of crossed polarizers as outlined in Fig. 2(a), the transmitted light intensity will strongly depend on both the orientation of the weave with respect to the polarizer system as well as $\varphi$. Since such a train of optical elements behaves in a non-commutative manner, we can expect their appearance to vary for different sequences of the two tapes. 
(a)

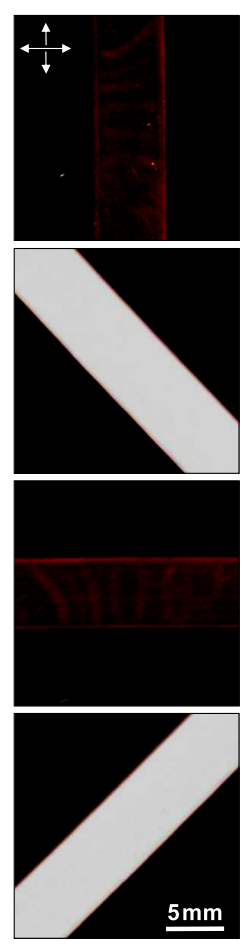

(b)
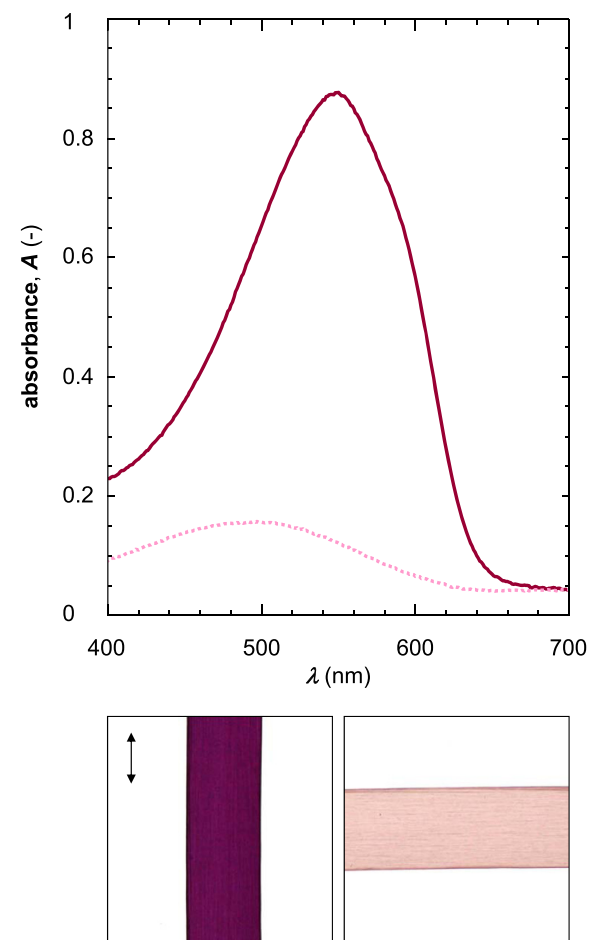

FIG. 1. Dichroism of tensile-drawn poly(3-hexylthiophene)/ultra-high molecular-weight polyethylene (P3HT/UHMW-PE) tapes. (a) Crosspolarized photographs of tapes (orientation of crossed polarizers as indicated). Note the high level of birefringence (variation in light intensity) as well as dichroism (change in color) of this system. (b) UV-Vis absorbance spectra (top panel) and photographs (bottom panel) of a tape backilluminated with light polarized parallel (solid line) and perpendicular (dashed line) to the drawing direction (orientation of polarizer as indicated).

This prediction could be confirmed experimentally for tapes plaited at $\varphi= \pm 45^{\circ}$ as displayed in Figs. 2(b)-2(d). Cross-polarized optical micrographs of two adjacent segments reveal that in one configuration the element with $\varphi_{\mathrm{A}} \sim+45^{\circ}$ appears dark purple when the system is backilluminated with white light, whereas the reverse weaving sequence, $\varphi_{\mathrm{B}} \sim-45^{\circ}$, gives rise to a pale pink color (Fig. 2(b)). Interestingly, the appearance of such pixels is similar to that of individual tapes when examined with a single polarizer (cf. Fig. 1(b)). Moreover, as expected, the absorbance only differs for wavelengths between 450 and $650 \mathrm{~nm}$, at which the oriented P3HT moiety gives rise to polarization dependent attenuation of the transmitted light (Fig. 2(d)). More generally, we observe similar position-dependent variation in light transmittance as long as $\varphi \neq \pm 90^{\circ}$, for which, in stark contrast, the appearance of adjacent tapes is little affected at different angles of orientation. ${ }^{26}$ Of course, a second exception is that of parallel stacks, i.e., $\varphi= \pm 0^{\circ}$, because in this case, varying the plaiting order will leave the structure unaffected, and, in addition, is highly unfeasible as this configuration cannot result in coherent fabrics.

Encouraged by the rich optical behavior that can be obtained with single weaves of linearly birefringent as well as dichroic elements, we went on to study the appearance of a more extended fabric produced with the present P3HT/ UHMW-PE tapes. Here, we chose to study the most straightforward case of plain weave fabrics woven with $\varphi \sim \pm 45^{\circ}$,

(a)

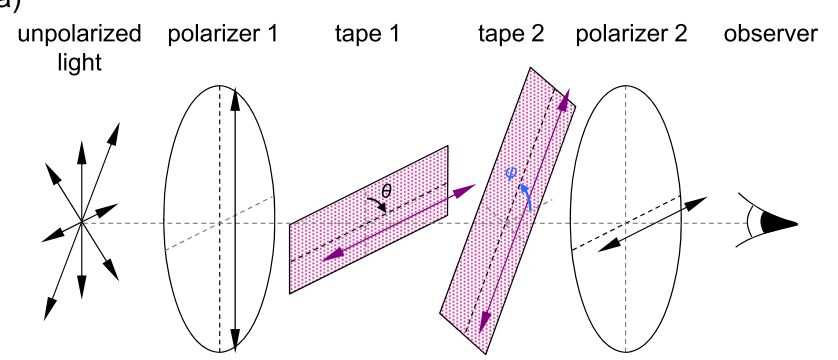

(b)

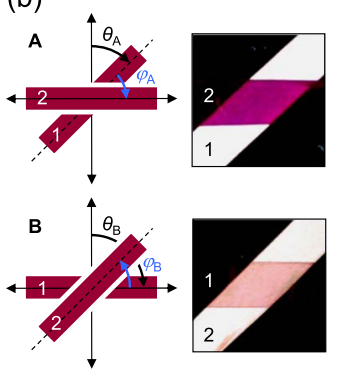

(c)

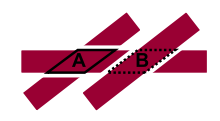

(d)

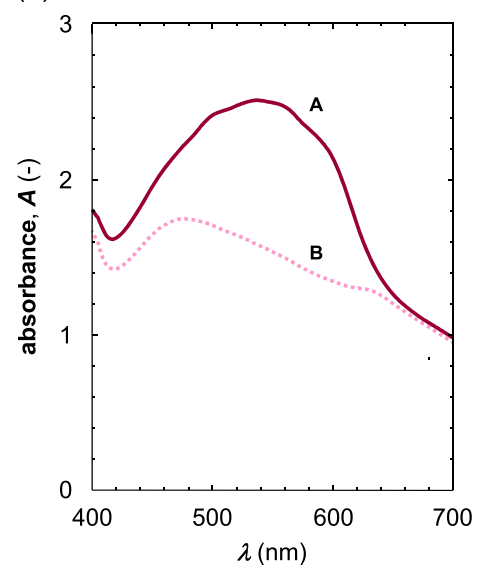

FIG. 2. Optical appearance of a single weave. (a) Setup used to study the optical appearance of a single weave of two P3HT/UHMW-PE tapes sandwiched between stationary crossed polarizers. The drawing direction of tape 1 is oriented at angle $\theta$ with respect to polarizer 1 , whereas tape 2 is oriented at angle $\theta+\varphi$, where angle $\varphi$ describes the relative angle between the two tapes. (b) Schematics (left) and cross-polarized photographs (right) of plaited P3HT/UHMW-PE tapes (orientation of crossed polarizers and stacking order of tapes 1 and 2 as indicated) overlapping at $\varphi_{\mathrm{A}} \sim+45^{\circ}$ (A) and $\varphi_{\mathrm{B}} \sim-45^{\circ}$ (B). (c) Schematic of adjacent segments A and B of a basketcase like alternating weaving pattern. (d) Cross-polarized UV-vis absorbance spectra of adjacent segments A and B. This measurement was performed with a Perkin Elmer Lambda 900 spectrophotometer equipped with a pair of crossed polarizers.

as discussed above. Indeed, a section of such a plain weave fabric produced with our P3HT/UHMW-PE tapes featured a checkered pattern of alternating bright yellow and dark purple pixels as revealed by cross-polarized photographs in Fig. 3(a). The contrast between adjacent pixels varied depending on the orientation of the fabric with respect to the stationary polarizer system. For instance, maximum contrast was observed at, $\sim 65^{\circ}$ and $\sim 155^{\circ}$ of rotation, whereas at $\sim 110^{\circ}$ and $\sim 205^{\circ}$ the fabric featured a largely uniform appearance.

The optical performance of such an optical system can be mathematically described by Müller calculus. ${ }^{27}$ In transmittance, each weave, if viewed between crossed polarizing sheets, can be regarded as a train of two perpendicular polarizers that sandwich two linearly birefringent as well as linearly dichroic elements (cf. Fig. 2(a)). The incident, unpolarized light of intensity $I_{0}$ is represented by the Stokes vector, $S_{0}$, with $S_{0}^{T}=\left(I_{0}, 0,0,0\right){ }^{28}$ According to Müller calculus, the transmitted intensity of a weave is then given by the first component of the Stokes vector, $S$

$$
S=M_{\text {polarizer2 }} \cdot M_{\text {tape } 2} \cdot M_{\text {tape1 }} \cdot M_{\text {polarizer } 1} \cdot S_{0}
$$

The following Müller matrices were used for a linear polarizer oriented at angle $\alpha^{29}$ and for a linearly birefringent as 
(a)
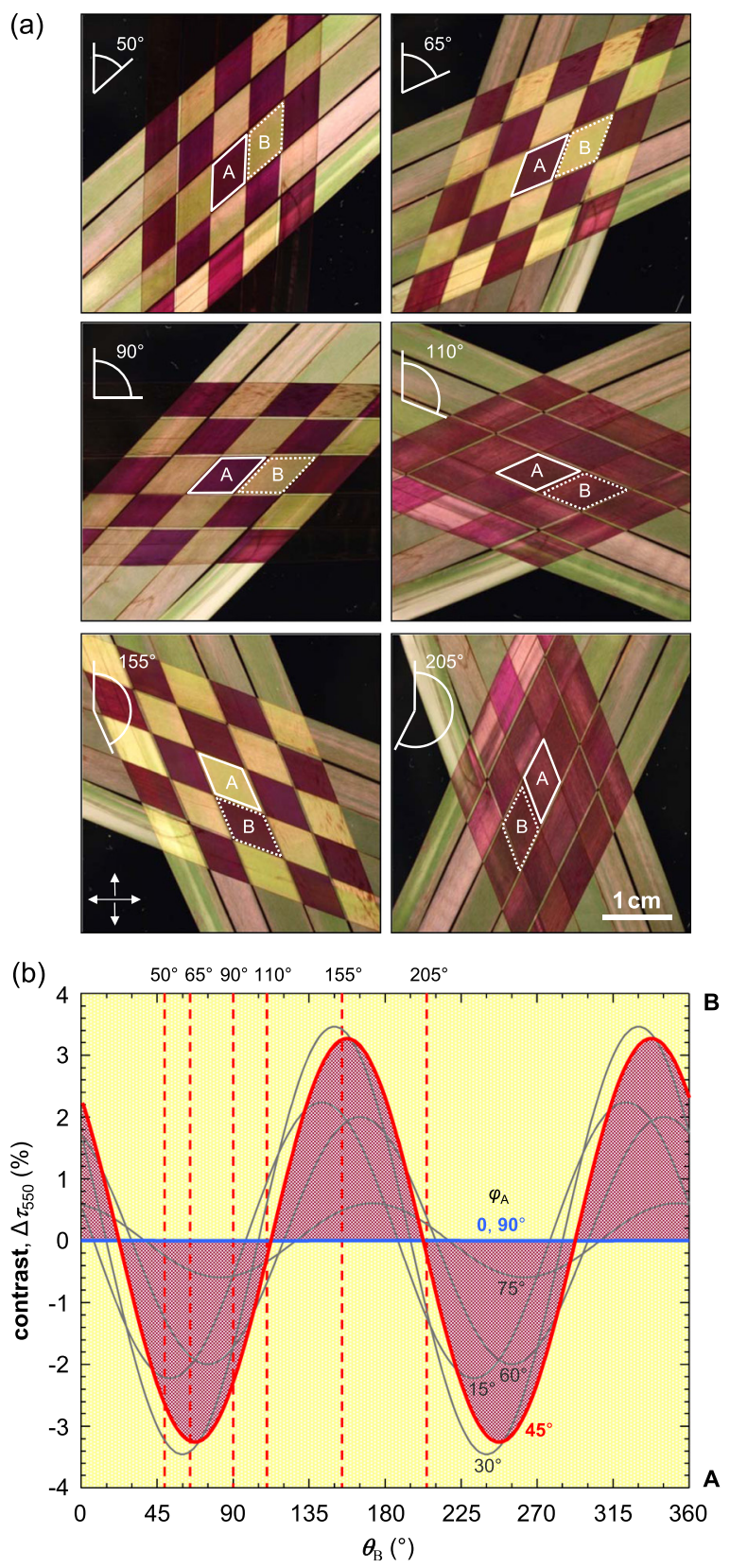

FIG. 3. Optical appearance of a plain weave fabric. (a) Cross-polarized photographs of a plain weave fabric $\left(\varphi= \pm 45^{\circ}\right)$ at various orientations with respect to the stationary polarizer system; two adjacent weaves, $\mathrm{A}$ and $\mathrm{B}$, are marked in each photograph (orientation of the fabric and the crossed polarizers as indicated; the yellow appearance of bright pixels results from the non-white backlight used to record these images). (b) Calculated variation in contrast at $550 \mathrm{~nm}, \Delta \tau_{550}=\tau_{\mathrm{A}}-\tau_{\mathrm{B}}$, between adjacent pixels of fabrics plaited at $\varphi= \pm 0^{\circ}, \pm 15^{\circ}, \pm 30^{\circ}, \pm 45^{\circ}, \pm 60^{\circ}, \pm 75^{\circ}$, and $\pm 90^{\circ}$ when rotating the fabric with respect to the stationary polarizer system. The red shaded curve corresponds to a fabric with $\varphi= \pm 45^{\circ}: \Delta \tau_{550}<0$ if A appears darker than $\mathrm{B}$ and $\Delta \tau_{550}>0$ if $\mathrm{A}$ appears brighter than $\mathrm{B}$. The angles of rotation that correspond to the photographs in (a) are indicated with dashed lines.

well as linearly dichroic element, e.g., a stretched P3HT/ UHMW-PE tape, oriented at angle $\theta^{30}$

$$
M_{\text {polarizer }}=\frac{1}{2} \cdot\left[\begin{array}{cccc}
1 & c_{2 \alpha} & s_{2 \alpha} & 0 \\
c_{2 \alpha} & c_{2 \alpha}^{2} & s_{2 \alpha} c_{2 \alpha} & 0 \\
s_{2 \alpha} & s_{2 \alpha} c_{2 \alpha} & s_{2 \alpha}^{2} & 0 \\
0 & 0 & 0 & 0
\end{array}\right],
$$

$$
\begin{aligned}
& M_{\text {tape }} \\
& =e^{\tilde{A}} \cdot\left[\begin{array}{cccc}
C_{\delta^{\prime}} & -c_{2 \theta} S_{\delta^{\prime}} & -s_{2 \theta} S_{\delta^{\prime}} & 0 \\
-c_{2 \theta} S_{\delta^{\prime}} & c_{2 \theta}^{2} C_{\delta^{\prime}}+s_{2 \theta}^{2} c_{\delta} & s_{2 \theta} c_{2 \theta}\left(C_{\delta^{\prime}}-c_{\delta}\right) & -s_{2 \theta} s_{\delta} \\
-s_{2 \theta} S_{\delta^{\prime}} & s_{2 \theta} c_{2 \theta}\left(C_{\delta^{\prime}}-c_{\delta}\right) & s_{2 \theta}^{2} C_{\delta^{\prime}}+c_{2 \theta}^{2} c_{\delta} & c_{2 \theta} s_{\delta} \\
0 & s_{2 \theta} s_{\delta} & -c_{2 \theta} s_{\delta} & c_{\delta}
\end{array}\right],
\end{aligned}
$$

where $s_{2 \alpha}=\sin 2 \alpha, c_{2 \alpha}=\cos 2 \alpha, s_{2 \theta}=\sin 2 \theta, c_{2 \theta}=\cos 2 \theta$, $s_{\delta}=\sin \delta, c_{\delta}=\cos \delta, S_{\delta^{\prime}}=\sinh \delta^{\prime}$, and $C_{\delta^{\prime}}=\cosh \delta^{\prime} .{ }^{31}$ The tape features a mean absorbance, $\tilde{A}$

$$
\tilde{A}=\frac{\ln 10}{2} \cdot\left(A_{x}+A_{y}\right),
$$

where $A_{\mathrm{x}}$ and $A_{\mathrm{y}}$ are the absorbance parallel and perpendicular to the orientation direction of the tape, a retardance, $\delta$

$$
\delta=\frac{2 \pi \cdot \Delta n \cdot d}{\lambda},
$$

where $\Delta n$ is the birefringence and $d$ the thickness of the tape, and an attenuation, $\delta^{\prime 32}$

$$
\delta^{\prime}=\frac{\ln 10}{2} \cdot\left(A_{x}-A_{y}\right) .
$$

The linear dichroism of P3HT/UHMW-PE tapes could be conveniently estimated using polarized UV-Vis absorbance spectroscopy. For instance, at $550 \mathrm{~nm}$ we observe a maximum absorbance, $A_{\mathrm{x}, 550} \sim 0.88$, parallel to the orientation direction of the tape and a much reduced perpendicular absorbance, $A_{\mathrm{y}, 550} \sim 0.12$ (cf. Fig. 1(b)). Thus, using Eqs. (4) and (6) we obtain $\tilde{A}_{550} \sim 1.151$ and $\delta_{550}^{\prime} \sim 0.875$.

The linear birefringence of highly oriented polyethylenes is insensitive to the exact draw ratio and thus we estimate a value of $\Delta n \sim 0.058$ according to Ref. $33 .{ }^{26} \mathrm{We}$ assume a largely wavelength-independent birefringence and negligible contribution from the $\mathrm{P} 3 \mathrm{HT}$ guest species and employ Eq. (5) to obtain a retardance $\delta_{550} \sim 6.626$ at $550 \mathrm{~nm}$ for a thickness $d=10 \mu \mathrm{m}$.

Employing these values for $\delta_{550}, \delta_{550}^{\prime}$, and $\tilde{A}_{550}$, we subsequently calculated the transmittance of weaves $\mathrm{A}$ and $\mathrm{B}$, and using those the difference in transmittance at $550 \mathrm{~nm}$, $\Delta \tau_{550}=\tau_{\mathrm{A}}-\tau_{\mathrm{B}}$, between two adjacent weaves for a full rotation of the fabric between stationary polarizing sheets (Fig. $3(\mathrm{~b})) .{ }^{26}$ It is immediately apparent that tapes plaited at $\varphi= \pm 0^{\circ}$ as well as $\varphi= \pm 90^{\circ}$ are confirmed to yield little to no contrast. Gratifyingly, however, our calculations suggest that intermediate weaving angles and in particular $\varphi= \pm 15^{\circ}$ to $\pm 60^{\circ}$ can be anticipated to give rise to an appreciable variation in the amount of transmitted light. According to our calculations, maximum contrast can be expected for $\varphi \sim \pm 35^{\circ} .{ }^{26}$ In addition, we find that the contrast of the two weaves will change sign every quarter rotation, implying that their appearance will alternate accordingly. In agreement with our experimental observations, for $\varphi \sim \pm 45^{\circ}$, maximum contrast was found to occur at $68^{\circ}$ and $158^{\circ}$ of rotation, and zero contrast at $113^{\circ}$ and $203^{\circ}$. Evidently, both the contrast between differently colored pixels as well as the periodic variation in light transmittance when rotating the 
system between stationary crossed polarizers could be accurately described by taking into account the linear birefringence arising from the uniaxially drawn polyethylene matrix as well as the linear dichroism caused by the orientation of the P3HT guest moiety.

Eventually, we could utilize the judicious alteration of the weaving sequence-i.e., selective interlacing of weft yarns into the warp layer ${ }^{20}$ - to fabricate intricate patterns with the present P3HT/UHMW-PE tapes (Fig. 4). Interestingly, the resulting image was readily apparent when investigated between crossed polarizers but not when viewed in unpolarized light. Thus, clearly, the here demonstrated woven systems permit facile production of concealed optical features in seemingly uniform fabrics.
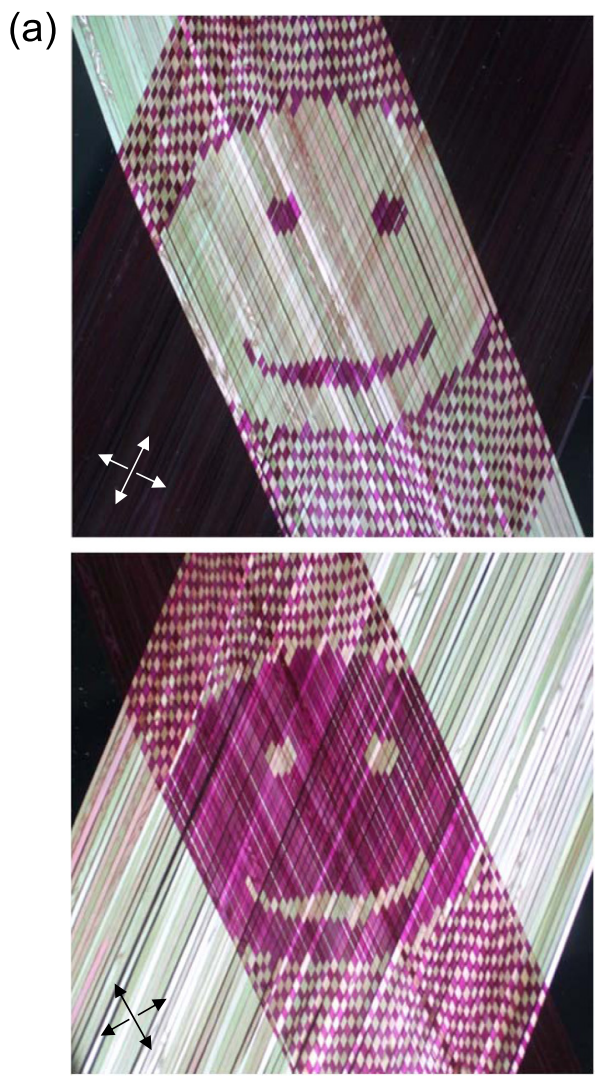

(b)

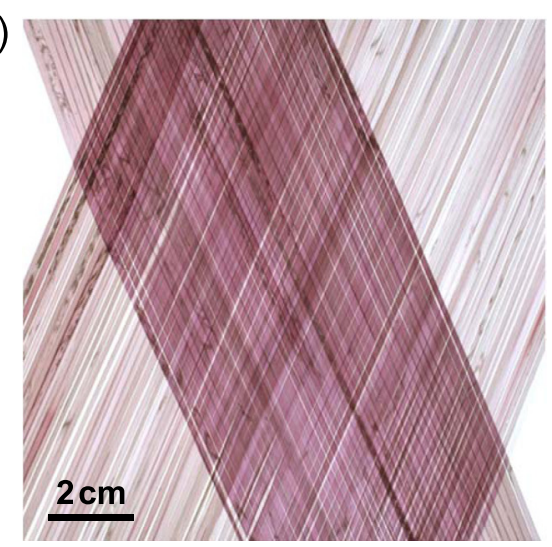

FIG. 4. Concealed image in an extended fabric. (a) Cross-polarized photographs of a fabric produced with P3HT/UHMW-PE tapes woven at $\varphi \sim \pm 45^{\circ}$, illustrating the potential for incorporation of concealed optical elements in textile structures (orientation of crossed polarizers as indicated). (b) Unpolarized photograph of the same fabric, displaying a uniform, featureless appearance when observed with white backlight.
Without doubt, we have shown that weaving of discrete optical elements is a versatile patterning technique that is readily applicable to established textile manufacturing routines. In principle, plaited structures similar to the ones presented here can be realized using a wide range of optically anisotropic tapes or fibers. Besides polyethylene, other suitable matrix polymers are for instance poly(ethylene oxide), polyamides or poly(vinyl alcohol), which can accommodate more polar guest species. A wide variety of other dye molecules could be used as long as they feature (i) a high aspect ratio to facilitate efficient uniaxial alignment and (ii) a transition dipole moment that largely coincides with the long axis of the molecule. ${ }^{34}$ Moreover, we anticipate that the concept explored in this letter can be applied to other advanced optoelectronic characteristics such as, among others, (polarized) photo- and electroluminescence or electrochromism, for use in flexible display and smart textile applications.

We are deeply indebted to Dr. Natalie Stingelin (Imperial College London) and Professor Paul Smith (ETH Zürich) for their generous support and for providing the unique, thought-inspiring environment of Geordies' M.C.-Q. and M.G. acknowledge the Ministerio de Economía y Competitividad for funding through Project No. PLE2009-0086.

${ }^{1}$ P. Dyreklev, M. Berggren, O. Inganäs, M. R. Andersson, O. Wennerström, and T. Hjertberg, Adv. Mater. 7, 43 (1995).

${ }^{2}$ M. Hamaguchi and K. Yoshino, Appl. Phys. Lett. 67, 3381 (1995).

${ }^{3}$ K. S. Whitehead, M. Grell, D. D. C. Bradley, M. Jandke, and P. Strohriegl, Appl. Phys. Lett. 76, 2946 (2000).

${ }^{4}$ D. Sainova, A. Zen, H. G. Nothofer, U. Asawapirom, U. Scherf, R. Hagen, T. Bieringer, S. Kostromine, and D. Neher, Adv. Funct. Mater. 12, 49 (2002).

${ }^{5}$ P. Andersson, M. Berggren, and T. Kugler, Appl. Phys. Lett. 83, 1307 (2003).

${ }^{6}$ C. Weder, C. Sarwa, C. Bastiaansen, and P. Smith, Adv. Mater. 9, 1035 (1997).

${ }^{7}$ A. Montali, C. Bastiaansen, P. Smith, and C. Weder, Nature 392, 261 (1998).

${ }^{8}$ M. Eglin, A. Montali, A. R. A. Palmans, T. Thervoort, P. Smith, and C. Weder, J. Mater. Chem. 9, 2221 (1999).

${ }^{9}$ Y. Cao, N. Colaneri, A. J. Heeger, and P. Smith, Appl. Phys. Lett. 65, 2001 (1994).

${ }^{10}$ C. Weder and P. Smith, U.S. patent 7,108,286 (Sept. 18, 2006).

${ }^{11}$ C. Weder, C. Sarwa, A. Montali, G. Bastiaansen, and P. Smith, Science 279, 835 (1998).

${ }^{12}$ E. H. Land, J. Opt. Soc. Am. 41, 957 (1951).

${ }^{13}$ T. W. Hagler, K. Pakbaz, J. Moulton, F. Wudl, P. Smith, and A. J. Heeger, Polym. Commun. 32, 339 (1991).

${ }^{14}$ C. Bastiaansen, H. W. Schmidt, T. Nishino, and P. Smith, Polymer 34, 3951 (1993).

${ }^{15}$ Y. Dirix, T. A. Tervoort, and C. Bastiaansen, Macromolecules 28, 486 (1995).

${ }^{16}$ C. Kocher, A. Montali, P. Smith, and C. Weder, Adv. Funct. Mater. 11, 31 (2001).

${ }^{17}$ C. Kocher, C. Weder, and P. Smith, Appl. Opt. 42, 5684 (2003).

${ }^{18}$ C. Müller, S. Goffri, D. W. Breiby, J. W. Andreasen, H. D. Chanzy, R. A. J. Janssen, M. M. Nielsen, C. P. Radano, H. Sirringhaus, P. Smith, and N. Stingelin-Stutzmann, Adv. Funct. Mater. 17, 2674 (2007).

${ }^{19}$ M. Campoy-Quiles, Y. Ishii, H. Sakai, and H. Murata, Appl. Phys. Lett. 92, 213305 (2008).

${ }^{20} \mathrm{H}$. Eberle and R. Kilgus, Clothing Technology (Verlag Europa-Lehrmittel Nourney, Vollmer GmbH \& Co KG, 2008).

${ }^{21}$ O. Inganäs, W. R. Salaneck, J.-E. Österholm, and J. Laakso, Synth. Met. 22, 395 (1988).

${ }^{22}$ R. D. McCullough, R. D. Lowe, M. Jayaraman, P. C. Ewbank, and D. L. Anderson, Synth. Met. 55, 1198 (1993).

${ }^{23}$ T.-A. Chen, X. Wu, and R. D. Rieke, J. Am. Chem. Soc. 117, 233 (1995). 
${ }^{24}$ H. Heil, T. Finnberg, N. von Malm, R. Schmechel, and H. von Seggern, J. Appl. Phys. 93, 1636 (2003).

${ }^{25}$ P. Parkinson, C. Müller, N. Stingelin, M. B. Johnston, and L. M. Herz, J. Phys. Chem. Lett. 1, 2788 (2010).

${ }^{26}$ See supplementary material at http://dx.doi.org/10.1063/1.4764518 for optical appearance of tapes woven at $\varphi_{\mathrm{B}} \sim \pm 90^{\circ}$; estimate of the birefringence; calculation of the contrast between two adjacent weaves of opposite stacking sequence; and a plot of the maximum contrast as a function of $\varphi_{\mathrm{A}}$.

${ }^{27}$ H. Müller, J. Opt. Soc. Am. 38, 661 (1948).
${ }^{28}$ G. G. Stokes, Trans. Cambrigde Philos. Soc. 9, 399 (1852).

${ }^{29}$ Polarizer 1 is fixed at $\alpha=0^{\circ}$ and polarizer 2 at $\alpha=90^{\circ}$ (cf. Fig. 2(a)).

${ }^{30}$ The drawing direction of tape 1 is oriented at $\theta$ with respect to polarizer 1 , whereas tape 2 is oriented at $\theta+\varphi$ (cf. Fig. 2(a)).

${ }^{31}$ G. G. Fuller, Optical Rheometry of Complex Fluids (Oxford University Press, New York, 1995).

${ }^{32}$ J. Schellman and H. P. Jensen, Chem. Rev. 87, 1359 (1987).

${ }^{33}$ Y. J. L. Dirix, "Polarisers based on anisotropic absorbance or scattering of light," Ph.D. dissertation (TU/e, Eindhoven, 1997), p. 24.

${ }^{34}$ E. W. Thulstrup and J. Michl, J. Am. Chem. Soc. 104, 5594 (1982). 